Matthias Bächle*, Daniel Alexander Schwär, and Fernando Puente León

\title{
Analytic wavelet packets for robust ultrasonic flow measurement
}

\author{
Analytische Wavelet-Pakete zur robusten Ultraschall-Durchflussmessung
}

https://doi.org/10.1515/teme-2019-0093

Received June 25, 2019; accepted September 3, 2019

\begin{abstract}
A key element in robust transit-time ultrasonic flow measurement is the accurate estimation of the transittime difference. Conventional methods, such as crosscorrelation or the estimation in the phase domain, are limited in their robustness against signal distortions, interfering signals or noise. In this work, we present a novel method to estimate the transit-time difference through the fusion of selected analytic wavelet packet coefficients. The combination of the complex coefficients, which represent a projection of the signal on analytic wavelets, with a configurable time-frequency resolution allows a sub-sample estimation at the frequency of interest. After giving an introduction into the fundamentals of analytic wavelet packets based on multi-scale filtering, we introduce two features that correlate strongly with the transit-time difference. The selection and fusion of these features is done by using correlation coefficients with a calibration set and principal component analysis. Finally, using a clamp-on flow measurement system, the robustness against temperature variation and measurement noise is shown and compared with conventional methods.
\end{abstract}

Keywords: Time-frequency analysis, ultrasonic flow metering, analytic wavelet packets.

Zusammenfassung: Ein wichtiger Baustein zur robusten Ultraschall-Durchflussmessung, basierend auf dem Laufzeitdifferenzprinzip, ist die genaue Schätzung der Laufzeitdifferenz. Konventionelle Methoden, wie die Kreuzkorrelation oder die Schätzung im Phasenbereich, sind in ihrer Robustheit gegenüber Signalverzerrungen, Störsignalen oder Rauschen beschränkt. In dieser Arbeit präsentieren wir eine neue Methode zur Schätzung der Laufzeitdifferenz durch die Auswahl und Fusion von Koef-

\footnotetext{
*Corresponding author: Matthias Bächle, Karlsruhe Institute of Technology, Institute of Industrial Information Technology, Karlsruhe, Germany, e-mail: matthias.baechle@kit.edu, ORCID: https://orcid.org/0000-0001-7228-299X

Daniel Alexander Schwär, Fernando Puente León, Karlsruhe Institute of Technology, Institute of Industrial Information Technology, Karlsruhe, Germany, ORCID:

https://orcid.org/0000-0001-9639-7919 (D. A. Schwär)
}

fizienten aus analytischen Wavelet-Paketen. Die Kombination der komplexen Koeffizienten, die eine Projektion des Signals auf die analytischen Wavelets darstellen, mit einer einstellbaren Zeit-Frequenz-Auflösung erlaubt eine sub-sample-genaue Schätzung bei der relevanten Frequenz. Nach einer Einführung in die Grundlagen der auf Multiraten-Filterbänken basierenden analytischen Wavelet-Pakete führen wir zwei Merkmale ein, die stark mit der Laufzeitdifferenz korrelieren. Die Auswahl und Fusion dieser Merkmale wird mithilfe von Korrelationskoeffizienten an Kalibrierdaten und der Hauptkomponentenanalyse durchgeführt. Abschließend wird die Robustheit gegenüber Temperaturänderungen und Messrauschen an einem Clamp-on-Durchflussmesssystem gezeigt und mit den konventionellen Methoden verglichen.

Schlagwörter: Zeit-Frequenz-Analyse, Ultraschall-Durchflussmessung, analytische Wavelet-Pakete.

\section{Introduction}

Using the transit-time difference (TTD) of ultrasonic signals for flow measurement, as shown in Fig. 1, has many advantages, such as the possibility of a non-intrusive measurement, the non-existence of moving parts and the fact that no impurities in the fluids are necessary $[6,12]$. It is based on the principle that the waves sent in upstream direction propagate slower than those sent in downstream direction. The accuracy of transit-time ultrasonic flow measurements is mainly dependent on the estimation quality of the TTD [10], which, depending on the application, may need to be better resolved than the sampling time [12]. Therefore, classical methods for the TTD estimation, such as cross-correlation [1] or the calculation of the time interval between specific points, need to use an interpolation and work with a high precision [12]. These methods differ in their robustness against signal distortions, measurement noise, and additive interfering signals without measuring effect, which are caused by ultrasound solely propagating through the pipe walls. Especially the latter can cause high measurement errors [17] and needs 


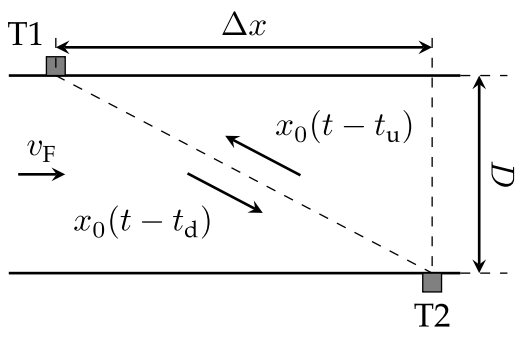

Figure 1: Principle of transit-time based ultrasonic flowmeter.

to be damped using constructive methods [11] or algorithmic approaches $[7,16]$. While the cross-correlation is robust against noise, it takes the whole signal into account and is therefore problematic when there is an additive interfering signal. In contrast, calculating the time interval between specific points such as zero-crossings allows a local information extraction, but is sensitive to noise. In order to combine the advantages of the different methods, our main objective was to find a method for TTD estimation that allows a compromise between local and global information extraction and uses only signal parts with a high robustness against both noise and interfering signals. For this purpose, we use wavelets with compact support for the analysis of the measurement signals, because of their tunable time-frequency resolution.

In the context of ultrasonic flow measurements, several methods to improve the TTD estimation are reported. Kupnik et al. [10] improved the robustness against noise and signal distortions by applying a linear fit in the phase domain, but the evaluation area was still limited to one period and the method is limited to narrow-band signals. A weighted version of the fit in the phase domain using the envelope as weights was presented by Roosnek [16], who also integrated a reduction of interfering signals by using flow statistics. Another possibility is to reduce the noise directly in a preprocessing step by the wavelet denoising method shown by Hua et al. [6]. Unlike the local TTD estimation using specific points, correlation based methods need to be combined with an interpolation to reach the desired sub-sample precision. This in turn corrupts the robustness against noise, what, as shown by Guetbi et al. [4], can be alleviated by relying on the cross-wavelet transform.

A different approach to estimate the TTD uses the shift theorem of the Fourier transform. It states that a shift in the time domain turns into a modulation in the frequency domain, which allows better time-delay resolutions, if the Nyquist-Shannon sampling theorem is satisfied. If the measurement signals have a narrow-band characteristic and the dominant frequency is known, the TTD can then be calculated from the phase shift between the upstream signal $x_{0}\left(t-t_{\mathrm{u}}\right)$ and the downstream signal $x_{0}\left(t-t_{\mathrm{d}}\right)$ by the equation

$$
\Delta t_{\text {est }}=\frac{1}{2 \pi f_{0}} \arg \left(\frac{\left.\mathcal{F}\left\{x_{0}\left(t-t_{\mathrm{d}}\right)\right\}\right|_{f=f_{0}}}{\left.\mathcal{F}\left\{x_{0}\left(t-t_{\mathrm{u}}\right)\right\}\right|_{f=f_{0}}}\right),
$$

with the estimated TTD $\Delta t_{\text {est }}$, the dominant frequency $f_{0}$ and the Fourier transform $\mathcal{F}\{\cdot\}$. However, applying a time window before the Fourier transform is necessary to take only signal parts with a measuring effect into account, leading to the short-time Fourier transform (STFT).

Time-frequency analysis methods, such as the STFT or the wavelet transform, are widely used in ultrasonic signal analysis. The range of application includes signal compression [15], classification [13], denoising [4], frequency or direction analysis in Doppler ultrasound systems $[9,19]$ and filtering [14]. In our approach, we pick up on the idea of using local frequency analysis to calculate the phase shift between the upstream and downstream signal. For this purpose, analytic wavelet packets (AWP) are used to analyze both the upstream and the downstream signal resulting in complex coefficients that describe the signals in a time-frequency domain. In this context, AWP, first introduced by Weickert et al. [22], represent an extension of real-valued wavelet packets to get complex base functions which build a Hilbert pair. Using this property, we can read the phase shift directly from the argument of the complex coefficients. In the next step, two features, namely the phase shift and the magnitude of the difference between the upstream and downstream coefficients, are calculated, due to their linear correlation with the TTD. In the last step, these features are weighted and fused to estimate the TTD.

The remainder of this paper is organized as follows. In Section 2, the fundamentals of the transformation, based on the AWP, are summarized. Then, the algorithm with all the necessary steps, such as the feature extraction, selection, and fusion, is described in Section 3. Finally, the robustness of the proposed method against noise, turbulence and interfering signals is validated using ultrasonic flow measurements at different temperature conditions and compared against the method proposed by Roosnek [16].

\section{Time-frequency analysis}

The objective of time-frequency analysis is the transformation of signals into a domain, where the local frequency 
components, which are changing over time, are observable. For time-discrete signals, this is equivalent to a decomposition

$$
x[n]=\sum_{s} \sum_{m} c_{s}[m] \psi_{s, m}[n],
$$

where the signal energy of the base function $\psi_{s, m}[n]$ is concentrated in a specific time range $t_{m}$ and frequency range $f_{s}$. Therefore, the coefficient $c_{s}[m]$ represents the frequency component $f_{s}$ of the signal $x[n]$ at the time $t_{m}$. If the base functions $\psi_{s, m}[n]$ are orthogonal, the coefficients $c_{s}[m]$ can be calculated by the inner product

$$
c_{s}[m]=\left\langle x[n], \psi_{s, m}[n]\right\rangle_{n} .
$$

In the literature, several approaches to design the base functions can be found. One method, called the discrete wavelet transform (DWT), is based on a multi-scale filtering and uses a cascaded filter bank, where each level consists of a low-pass filter $g_{\mathrm{LP}}[n]$ and a band-pass filter $g_{\mathrm{BP}}[n]$ with a subsequent downsampling by 2 (see the Daubechies wavelets [3] for further details on the filter design). The resulting base functions can be formulated as

$$
\psi_{s, m}[n]=2^{-s / 2} \psi\left[2^{-s} n-m\right],
$$

with the integer scale $s$, representing the number of levels in the cascade, and the time shift $m$ in samples.

Since the DWT works with downsampling, a timeshifted signal can lead to a completely different result in the time-frequency domain. Another problem of the real DWT is the oscillation of the resulting coefficients even if the signal energy in the corresponding frequency range is constant. This effect can be explained by the phase dependency of the inner product between real-valued harmonic signals. An extension of the DWT to enable a timefrequency analysis with complex base functions, comparable to the Fourier transform, is realized by an additional tree for the imaginary part of the base functions. The base function of the imaginary tree $\psi_{s, m}^{\mathrm{Im}}[n]$ and the real tree $\psi_{s, m}^{\mathrm{Re}}[n]$ need to build a Hilbert pair, so that the complex base function

$$
\psi_{s, m}[n]=\psi_{s, m}^{\mathrm{Re}}[n]+\mathrm{j} \psi_{s, m}^{\operatorname{Im}}[n]
$$

is an analytical signal. This is important to lessen the aforementioned oscillations of the resulting coefficients and to improve the translation invariance of the transformation. The resulting complex coefficients can then be calculated by

$$
\begin{aligned}
c_{s}[m] & =\left\langle x[n], \psi_{s, m}[n]\right\rangle_{n} \\
& =\left\langle x[n], \psi_{s, m}^{\mathrm{Re}}[n]+\mathrm{j} \psi_{s, m}^{\mathrm{Im}}[n]\right\rangle_{n} \\
& =c_{s}^{\mathrm{Re}}[m]+\mathrm{j} c_{s}^{\mathrm{Im}}[m] .
\end{aligned}
$$

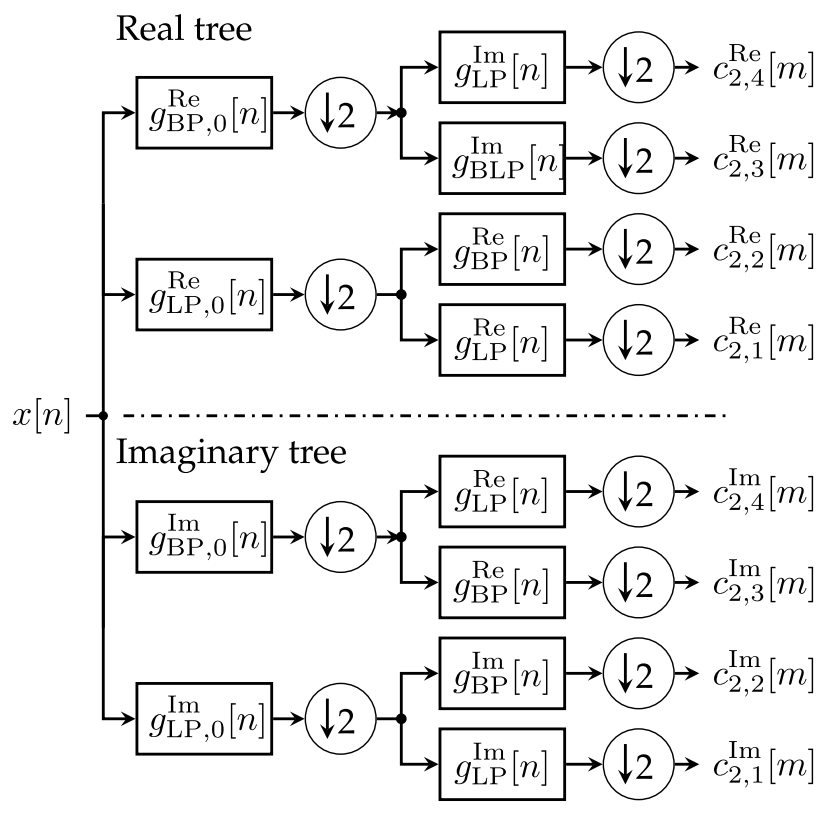

Figure 2: Multi-scale filter bank for the AWP.

This method, called the dual-tree complex wavelet transform (DT-CWT), was described by Selesnick et al. [18].

For the analysis of ultrasonic signals, using the standard wavelet bases with high frequency resolution for lower frequencies may not yield the best representation and is not adaptable enough. Therefore, in this work, the measurement signals of the ultrasonic transducers are analyzed using the AWP (see Fig. 2 for an example with two levels). Compared to the DT-CWT, the band-pass signals are also split up further, resulting in a full tree with a special filter arrangement, as explained in [22]. Note that the notation of the resulting coefficients $c_{s, k}^{\mathrm{Re}}[m]$ and $c_{s, k}^{\mathrm{Im}}[m]$ in Fig. 2 is extended to contain the AWP scale $s$ and the frequency sub-band $k$. For the application of the AWP, the free parameters scale $s$, filter types and filter length need to be set. As described in Selesnick et al. [18], the first level filters have to meet different requirements than the subsequent filters in order to get analytical base functions. For the first level filters the relation

$$
g_{\mathrm{LP}, 0}^{\mathrm{Im}}[n]=g_{\mathrm{LP}, 0}^{\mathrm{Re}}[n-1]
$$

has to be fulfilled, which can be realized through the Daubechies-10 wavelets [3] and a shift by one sample. For the subsequent filters the so called half-sample-delay condition

$$
g_{\mathrm{LP}}^{\mathrm{Im}}[n]=g_{\mathrm{LP}}^{\mathrm{Re}}[n-0.5]
$$

has to hold. Therefor a special type of filter, which is conform with (8), has to be used, such as the Q-shift-14 
wavelets [8]. The lengths of both filters are chosen considering the steepness of their frequency response and the similarity of the resulting base functions with the measurement signals. The choice of the scale $s$ is dependent on the frequency range of interest and the sampling rate. Under the assumption that the frequency band is equally split at each level and the signal $x[n]$ has half the sample rate $f_{\mathrm{s}}$ as frequency resolution according to the Nyquist-Shannon sampling theorem, the scale-dependent frequency resolution $\Delta f$ can be approximated by

$$
\Delta f=\frac{f_{\mathrm{s}}}{2^{s+1}} .
$$

This leads to $s \in[5,10]$ as reasonable choices, when the frequency of interest is between $500 \mathrm{kHz}$ and $1 \mathrm{MHz}$ and the sampling rate $f_{\mathrm{S}}=50 \mathrm{MHz}$ is used.

The result of the AWP, using the signal $x[n]$ as input, is a set of coefficients $c_{s, k}^{\mathrm{Re}}[m], c_{s, k}^{\mathrm{Im}}[m]$, which can be combined to complex-valued coefficients $c_{s, k}[\mathrm{~m}]$ using (6). Applying the AWP to a typical measurement signal (see Section 4) results in the time-frequency representations depicted in Fig. 3. It can be observed that the scale $s=6$ leads to a higher time resolution, whereas $s=8$ leads to a better frequency resolution. Because we only use coefficients on the same scale and to improve clarity, the index for the scale is omitted in the following section.

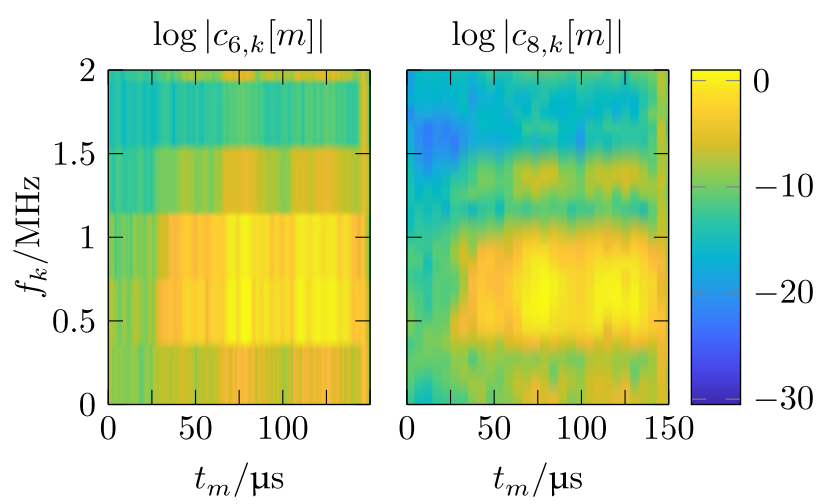

Figure 3: Different time-frequency representations resulting from the normalized magnitude of AWP coefficients of an example signal: scale $s=6$ (left) and $s=8$ (right).

\section{Robust estimation of the transit-time difference}

The principle of ultrasonic flow metering using TTD, as shown in Fig.1, can be explained as follows. An excita- tion signal is sent in both directions, upstream and downstream. Since the signals need a different time to propagate through the fluid, depending on the fluid velocity, the measurement model can be described as

$$
\begin{aligned}
& x_{\mathrm{u}}(t)=x_{0}\left(t-t_{\mathrm{u}}\right)+e_{\mathrm{u}}(t)+n_{\mathrm{u}}(t), \\
& x_{\mathrm{d}}(t)=x_{0}\left(t-t_{\mathrm{d}}\right)+e_{\mathrm{d}}(t)+n_{\mathrm{d}}(t),
\end{aligned}
$$

with the direct propagation signal $x_{0}(t)$, the electronic noise $n(t)$, and the various deterministic effects like reflections or interfering signals $e_{\mathrm{u}}(t), e_{\mathrm{d}}(t)$. In this measurement model, the indices $u$ and $d$ denote upstream and downstream components, respectively. From the geometry of the setup with the axial distance $\Delta x$ and the speed of sound $c_{0}$, the fluid velocity

$$
v_{\mathrm{F}} \approx \frac{\Delta t c_{0}^{2}}{2 \Delta x}, \quad \Delta t=t_{\mathrm{u}}-t_{\mathrm{d}}
$$

can be derived approximately, if the relation $c_{0} \gg v_{\mathrm{F}}$ holds [6]. Further examination of (11) shows that the TTD at constant flow is dependent on the speed of sound $c_{0}$, which in turn is dependent on the temperature [5]. For example, assuming that water is used as medium and the flow is kept constant, an increasing temperature in the range $\left[20^{\circ} \mathrm{C}, 70^{\circ} \mathrm{C}\right]$ leads to a reduced absolute transit-time of the ultrasonic waves and a reduced TTD, because $c_{0}$ is increasing. It has to be noted that only the average velocity along the propagation path is calculated by (11). In order to get the real average velocity, the flow profile has to be considered by a correction factor, which is dependent on the Reynolds number [21].

\subsection{Feature extraction and calibration}

The measurement signals $x_{\mathrm{u}}[n], x_{\mathrm{d}}[n]$ are transformed by the AWP, as described in Section 2, to get a set of AWP coefficients $c_{k}^{\mathrm{u}}[m], c_{k}^{\mathrm{d}}[m]$, respectively. Now robust features have to be extracted which have a linear relationship with the TTD. Therefor, a set of features

$$
\mathcal{F}=\left\{\left|c_{k}^{\mathrm{u}}[m]-c_{k}^{\mathrm{d}}[m]\right|, \arg \left(\frac{c_{k}^{\mathrm{u}}[m]}{c_{k}^{\mathrm{d}}[m]}\right)\right\}, \quad \begin{aligned}
& k=1,2, \ldots, \\
& m=1,2, \ldots
\end{aligned}
$$

containing a magnitude feature and a phase feature for all the sub-bands $k$ and all the time shifts $m$, is calculated. Since the interfering signals are almost identical for the upstream and downstream direction, their influence is suppressed by calculating the magnitude of the signal difference. If the TTD is sufficiently small against the period duration of the dominant frequency, a linear relationship between the amplitude of the difference signal and 
the TTD is to be expected. Instead of subtracting the signals of each other and calculating the AWP afterwards, the order can be reversed and the time-frequency coefficients can be subtracted like in the magnitude feature presented in (12). This works due to the linearity of AWP and has the advantage that both features can be calculated at the same point. The second kind of feature in (12) is motivated by the shift theorem of the Fourier transform, as formulated in (1).

The elements of the feature set $\xi \in \mathcal{F}$ have to be evaluated regarding their linearity with the TTD. Therefore, a calibration set with $L$ measurements and the corresponding ground-truth data $\Delta \mathbf{t}=\left[\Delta t_{1}, \ldots, \Delta t_{L}\right]$ is needed. The $L$ measurements lead to $L$ realizations for every single feature $\xi$ in the feature set $\mathcal{F}$. These realizations and their ground-truth can be used to calculate the correlation coefficient

$$
r_{\Delta t, \xi}=\sum_{l=1}^{L} \frac{\left(\Delta t_{l}-\Delta \bar{t}\right) \cdot\left(\xi_{l}-\bar{\xi}\right)}{\sqrt{\sum_{l=1}^{L}\left(\Delta t_{l}-\Delta \bar{t}\right)^{2} \cdot \sum_{l=1}^{L}\left(\xi_{l}-\bar{\xi}\right)^{2}}}
$$

as an evaluation of the linearity with the TTD, for every feature $\xi$ in the feature set $\mathcal{F}$. Note that $(\cdot)$ denotes the arithmetic mean over the realizations. If the magnitude of the correlation coefficient is almost 1 , the feature has a linear relationship with the TTD, and due to this, is robust against interfering signals.

Figure 4 shows the magnitude of the correlation coefficients calculated by (13) in dependency of their corresponding time $t_{m}$ and frequency $f_{k}$. In this example, the calibration set which is presented in Section 4, is used. In comparison with Fig. 3, it can be seen that the highest correlation coefficients relate to the dominant frequency of the signal. Furthermore, the correlation coefficients increase at the arrival time of the direct propagation signal. The highest correlation coefficients in both feature maps

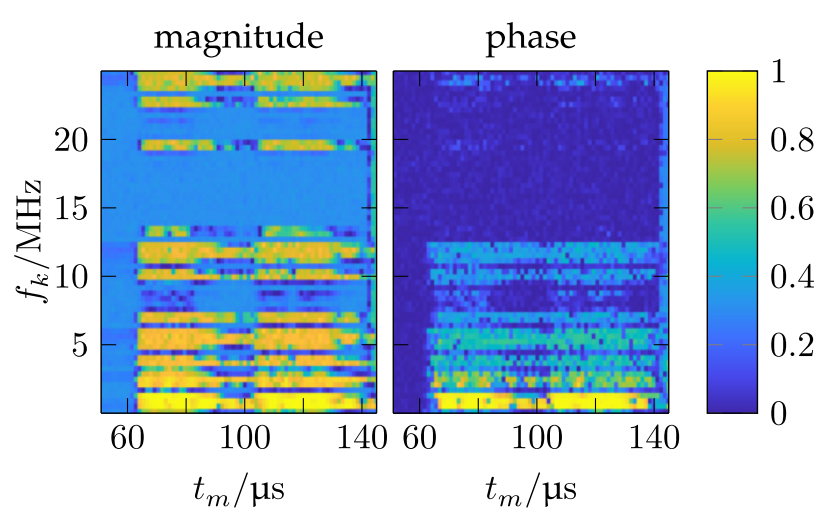

Figure 4: Correlation coefficients of the magnitude feature (left) and the phase feature (right). are almost equal to 1 , which confirms the choice of the features.

For the selection of robust features, a threshold $y$ has to be defined. Every feature whose correlation coefficient satisfies $\left|r_{\Delta t, \xi}\right| \geq \gamma$ is selected. Figure 5 shows the selection of the features for the threshold $y=0.995$, represented as a mask for the time-frequency domain of both features. It can be seen that all selected features are from similar branches, mostly at the absolute arrival times of the direct propagation path and the first reflection.

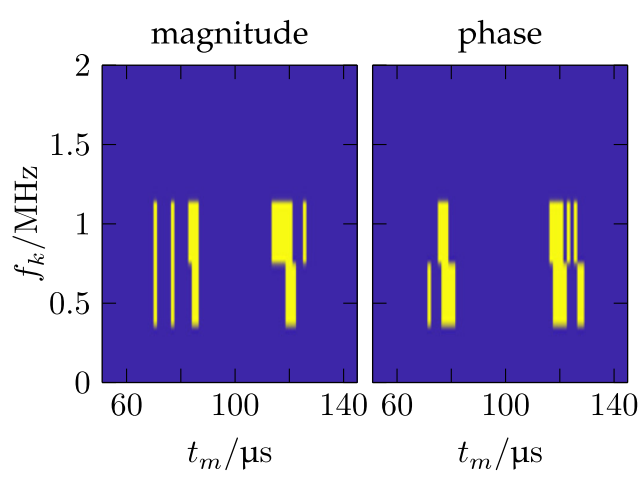

Figure 5: Mask of the selected features $(\gamma=0.995)$ : the magnitude feature (left) and the phase feature (right).

Suppose $P$ features were selected, then these features can be arranged with their $L$ realizations in the matrix

$$
\boldsymbol{\Xi}_{\mathrm{sel}}=\left[\begin{array}{ccc}
\xi_{1,1} & \ldots & \xi_{1, L} \\
\vdots & \ddots & \vdots \\
\xi_{P, 1} & \ldots & \xi_{P, L}
\end{array}\right]
$$

In order to build a relation between the features and the TTDs, the matrix $\boldsymbol{\Xi}_{\text {sel }}$ needs to be transformed by using a linear model.

$$
\mathbf{T}=\hat{\mathbf{A}} \Xi_{\text {sel }}+\hat{\mathbf{b}} \mathbf{I}^{\mathrm{T}}
$$

with

$$
\begin{aligned}
& \mathbf{I}^{\mathrm{T}}=[1, \ldots, 1] \in \mathbb{R}^{1 \times L}, \\
& \hat{\mathbf{A}}=\operatorname{diag}(\hat{\mathbf{a}}) \in \mathbb{R}^{P \times P}, \\
& \hat{a}_{p}=\operatorname{median}\left\{\frac{\Delta t_{l}-\Delta \overline{\mathbf{t}}}{\xi_{l, p}-\bar{\xi}_{p}}\right\}_{l}, \\
& \hat{b}_{p}=\Delta \overline{\mathbf{t}}-\hat{a}_{p} \cdot \bar{\xi}_{p} .
\end{aligned}
$$

Equation (15) can be interpreted as follows. For every feature, the model parameters $\hat{a}_{p}, \hat{b}_{p}$ of a separate linear model have to be estimated, which can be done by a leastsquares estimation or a median-based method. Since the 
least-squares estimation is susceptible to outliers, the median based method (18), (19) is preferred and listed in this method.

After the $P$ selected features are mapped onto the ground-truth vector $\Delta \mathbf{t}$, resulting in $P$ estimations of the TTD T, a fusion into a single improved estimation $\Delta t_{\text {est }}$ is necessary. To this end, two different methods were developed, which differ in their complexity and their robustness. Both methods deliver a weight vector $\mathbf{w}$, whereby a weighted arithmetic mean can be calculated. The first method, named the $\mathrm{AWP}_{\text {Max }}$, uses the feature with the maximum correlation coefficient, reducing the arithmetic mean to a maximum operation. For the second method, named $\mathrm{AWP}_{\mathrm{PCA}}$, the weight vector is calculated by the principal component analysis (see [2] for further reference). Therefor, the eigenvalue decomposition of the covariance matrix

$$
\operatorname{cov}(\mathbf{T})=(\mathbf{T}-E\{\mathbf{T}\})(\mathbf{T}-E\{\mathbf{T}\})^{\mathrm{T}}=\mathbf{V} \mathbf{\Lambda} \mathbf{V}^{\mathrm{T}}
$$

is calculated. With the first eigenvector $\mathbf{v}$, which represents the direction of the largest variance over the $L$ realizations, the weight vector is formed by the normalization

$$
\mathbf{w}=\frac{\mathbf{v}}{\sum_{p=1}^{P} v_{p}} .
$$

For future measurements, the model parameters $\hat{\mathbf{a}}, \hat{\mathbf{b}}$ and weight vector $\mathbf{w}$ can be combined. That forms the socalled fusion weights:

$$
\begin{aligned}
& \tilde{\mathbf{a}}=\mathbf{w}^{\mathrm{T}} \hat{\mathbf{A}}, \\
& \tilde{b}=\mathbf{w}^{\mathrm{T}} \hat{\mathbf{b}} .
\end{aligned}
$$

\subsection{Estimation algorithm}

After the selection of the features and their corresponding fusion weights, the second part of the AWP method performs the estimation of the TTD for new measurements. Because only a few features are chosen, as shown in Fig. 5, the AWP tree is simplified to calculate only the necessary branches, which in case of the $\mathrm{AWP}_{\mathrm{Max}}$ is only one branch. The coefficients of these branches are subsequently transformed into the feature space using (12) and arranged in the feature vector $\boldsymbol{\xi}_{\text {sel }}$. Alternatively, the whole tree can be calculated and transformed into the feature space, from where the necessary features are chosen according to the selection mask. Finally, the TTD can be estimated from the selected features by

$$
\Delta t_{\mathrm{est}}=\tilde{\mathbf{a}}^{\mathrm{T}} \boldsymbol{\xi}_{\mathrm{sel}}+\tilde{b} .
$$

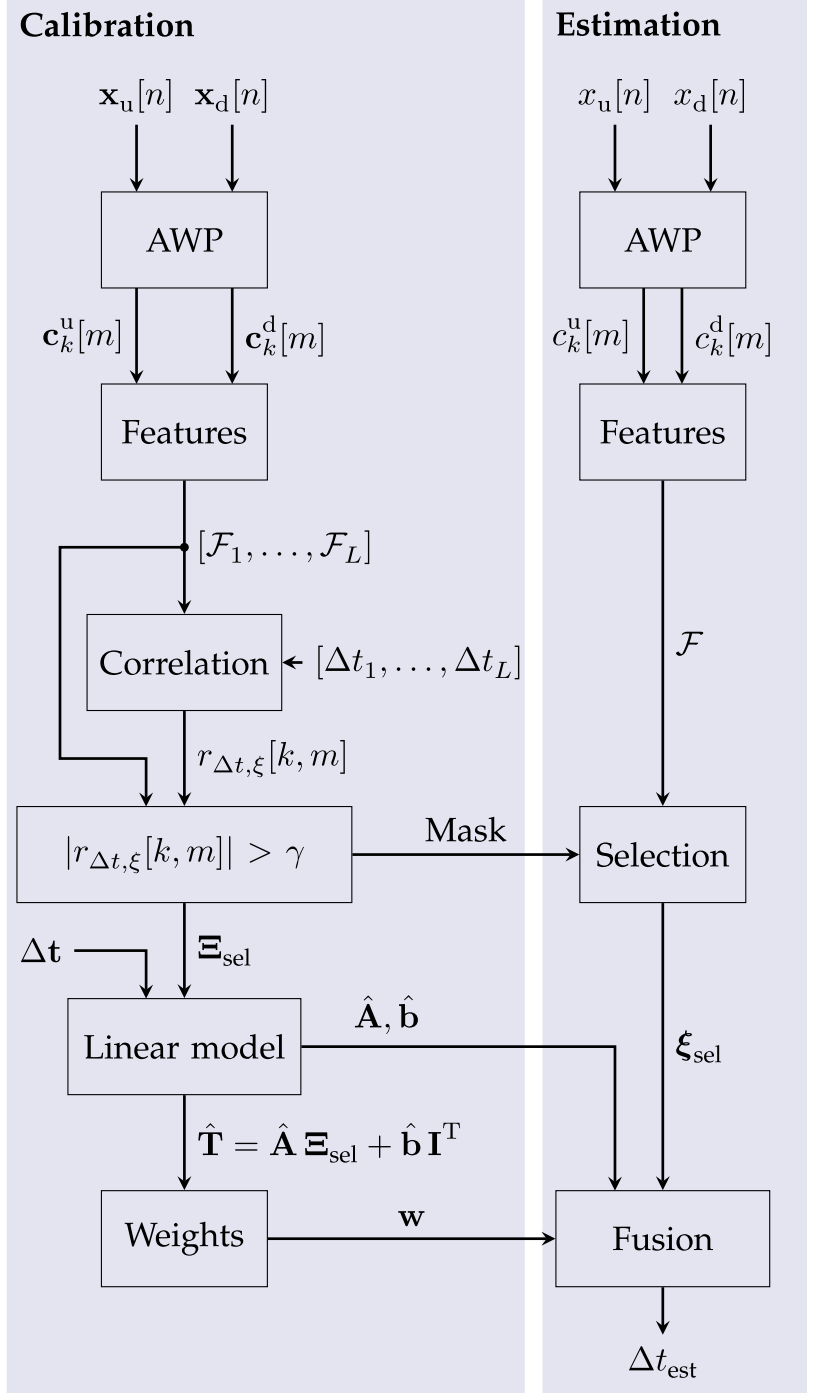

Figure 6: Flow chart of calibration and estimation algorithm.

Figure 6 depicts an overview of the AWP method in form of a flow chart. Summarized, the method can be separated in two parts, namely the calibration and the estimation part. In the calibration part, after the signals are analyzed by the AWP and transformed into the feature space $\mathcal{F}$, every feature is evaluated by the correlation coefficient $r_{\Delta t, \xi}$. In the next step, the features, whose correlation coefficients are exceeding the threshold $\gamma$, are mapped to a matrix $\mathbf{T}$ using a linear model (15). In the last step of the calibration part, the fusion weights are calculated by the combination of the model parameters and the weight vector using (22). Finally, the selection mask and the fusion weights are used in the estimation part for selecting the AWP coefficients, transforming them into the feature space and fusing them. 


\section{Experimental results}

For the validation of the proposed methods, which in the following are referred to as the AWP method with fused features $\left(\mathrm{AWP}_{\mathrm{PCA}}\right)$ and the AWP method with the best feature $\left(\mathrm{AWP}_{\mathrm{Max}}\right)$, two data sets, namely a calibration set $\mathcal{C}$ and a test set $\mathcal{T}$, were recorded from an ultrasonic flow measurement system with water as the medium (see Fig. 7). The pipe was made of stainless steel and was part of a circular flow with a pump to control flow, a heating element to control the temperature and a reference flowmeter to get the ground-truth TTDs. In order to get a fully developed turbulent flow profile, a flow straightener in combination with a sufficient inlet length was used.
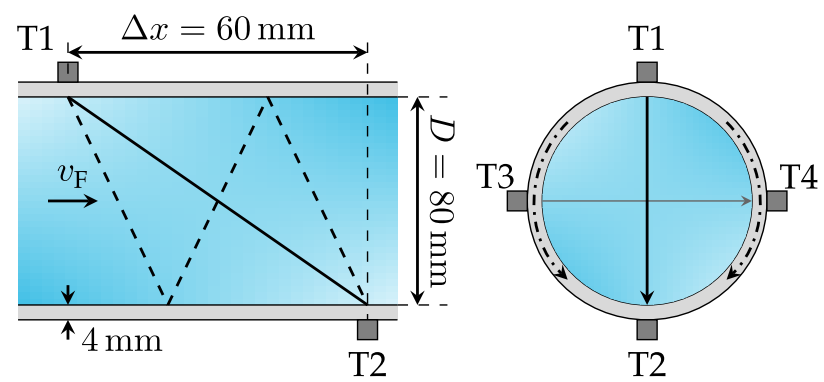

Figure 7: Ultrasonic flow measurement setup (not to scale). Three different propagation paths are shown: direct propagation (solid), first reflection (dashed) and interfering signals (dashdotted).

The data acquisition was performed at a sample rate of $f_{\mathrm{s}}=50 \mathrm{MHz}$ using a preamplifier and a PXIe-1062 station with a PXIe-5171 ADC module and a PXI-5412 DAC module, which also controlled the pump and the heating element.

As calibration set $\mathcal{C}$, we repeated a flow sequence

$$
v_{\mathrm{F}}[m]= \begin{cases}0.3 \mathrm{~m} / \mathrm{s} & m \in\{1,2, \ldots, 60\} \\ 0.6 \mathrm{~m} / \mathrm{s} & m \in\{61,62, \ldots, 100\} \\ 1.2 \mathrm{~m} / \mathrm{s} & m \in\{101,102, \ldots, 130\}\end{cases}
$$

19 times with different temperatures $T_{i} \in\left[19^{\circ} \mathrm{C}, 32.7^{\circ} \mathrm{C}\right]$ to cover the whole range of process parameters. As training set $\mathcal{T}$, we performed 1911 measurements, while the flow has been kept constant at $v_{\mathrm{F}}[\mathrm{m}]=1 \mathrm{~m} / \mathrm{s}$ and the temperature was increased from $19^{\circ} \mathrm{C}$ to $32.6^{\circ} \mathrm{C}$. Every measurement consists of one upstream and one downstream signal for each clamp-on transducer pair T1/T2 and T3/T4, which are arranged as shown in Fig. 7. Due to the pipe material, the geometric structure and the excitation signal

$$
u_{0}(t)=A \cdot \exp \left(-\frac{\left(t-t_{0}\right)^{2}}{2 \sigma^{2}}\right) \cdot \cos \left(2 \pi f_{0} t\right)
$$

with $A=10 \mathrm{~V}, t_{0}=5 \mu \mathrm{s}, \sigma=1.5 \mu \mathrm{s}$, and $f_{0}=700 \mathrm{kHz}$, the measurement signals contain a high level of interfering signals, the direct propagation signal and the first reflection signal.

On the basis of the calibration set and the test set, $\mathrm{AWP}_{\mathrm{PCA}}$ and $\mathrm{AWP}_{\mathrm{Max}}$ are compared with the TTD estimation that uses the absolute times of a fixed zero-crossing in the direct signal, as proposed by Roosnek [16]. Using the installed reference flowmeter and (11), we can calculate the ground-truth $\Delta t(T)$ for every temperature. Note that the temperature dependency of $c_{0}$ has to be considered, if (11) is used, whereas the flow profile can be neglected in this scope, as the relation between the features and the TTD is calibrated (see Section 3.1) and the same calibration values are used in the evaluation of the method. Thereby, the constant correction factor resulting from a constant flow is eliminated and the remaining error resulting from the variation of the Reynolds number between $20 \cdot 10^{3}$ and $130 \cdot 10^{3}$ during the calibration is approximately $0.5 \%$ [23]. The relative temperature-dependent measurement error

$$
E(T)=\frac{\Delta t_{\mathrm{est}}(T)-\Delta t(T)}{\Delta t(T)}
$$

is then used to evaluate the systematic error by the peakto-peak difference

$$
\Delta E_{\max }=\max _{T} E_{\mathrm{sys}}(T)-\min _{T} E_{\mathrm{sys}}(T)
$$

of the median filtered measurement error $E_{\text {sys }}(T)$. Furthermore, the robustness against noise and turbulence is investigated using the standard deviation of the measurement error after the systematic error has been corrected:

$$
\sigma_{\mathrm{E}}=\sqrt{\frac{1}{N-1} \sum_{n=1}^{N}\left(E\left(T_{n}\right)-E_{\text {sys }}\left(T_{n}\right)\right)^{2}} .
$$

In Fig. 8, the temperature dependency of the measurement error is shown for Roosnek's method, $\mathrm{AWP}_{\mathrm{Max}}$ and $\mathrm{AWP}_{\mathrm{PCA}}$, where both AWP methods use the whole calibration set and the AWP scale $s=6$. Firstly, the algorithms are evaluated on the same measurement set which has already been used for the calibration (Fig. 8a). It can be seen that the error is reduced significantly using AWP compared to Roosnek, even if the fusion part is omitted. Secondly, in Fig. 8b, the results on the test set $\mathcal{T}$ are shown to ensure that the estimation part of the AWP methods also works at different flow rates. Three conclusions can be drawn from Fig. 8b. Firstly, all three methods show a bad estimation quality when bubbles are present, which occurred due to some leftover air in our experimental stand that was randomly released and distributed by the pump 


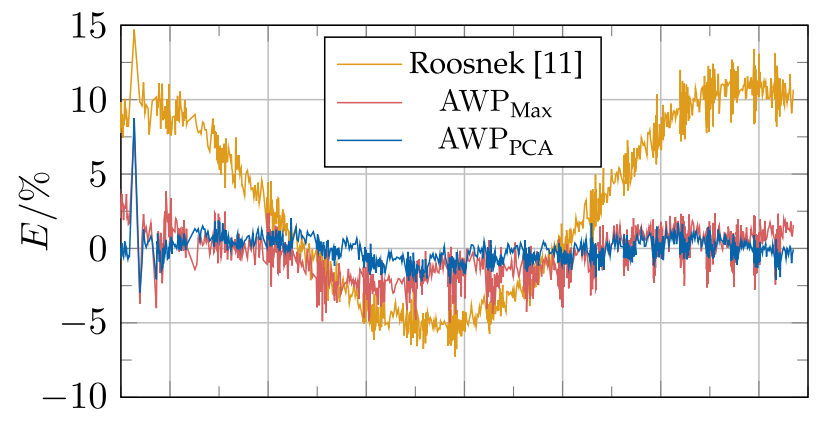

(a) Performance on calibration set $\mathcal{C}$ of $\mathrm{T} 1 / \mathrm{T} 2$

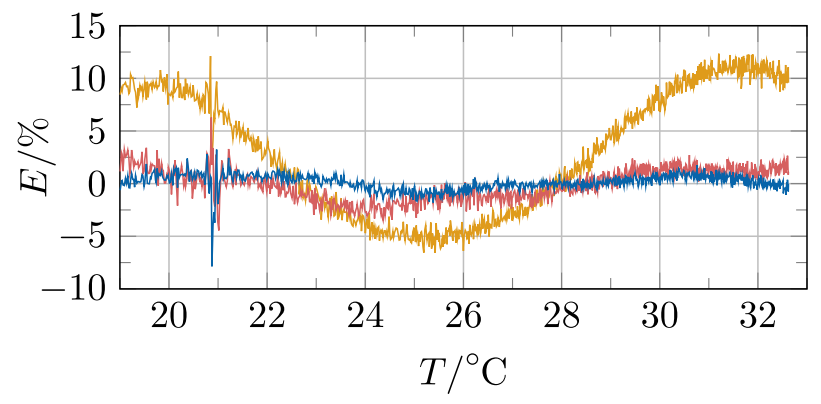

(b) Performance on test set $\mathcal{T}$ of $\mathrm{T} 1 / \mathrm{T} 2$.

Figure 8: Comparison of Roosnek's method with the AWP method using the whole calibration set $\mathcal{C}(d=6, \gamma=0.995)$ with PCA weights and without fusion.

(see at $T \approx 21^{\circ} \mathrm{C}$ ). Secondly, the systematic error, caused by the stationary interfering signals, is dependent on the temperature-varying phase shift between interfering signals and fluid signals and is represented by a slowly varying error. Due to the changing speed of sound and the geometry of the propagation path, the absolute transit-time is shifted by $1.60 \mu$ s during the temperature variation. Because one period takes $1.43 \mu \mathrm{s}$ at $f_{0}=700 \mathrm{kHz}$, it is expected that the measurement error of Roosnek's method shows a bit more than one oscillation in the temperature range under consideration, which is conform with the experimental results shown in Fig. 8b. This systematic error can be separated from the noise-induced fluctuation by using a median filter. Lastly, the $\mathrm{AWP}_{\mathrm{PCA}}$ is the best in terms of systematic error, closely followed by the $\mathrm{AWP}_{\text {Max }}$.

After the systematic error is removed by subtraction of the median filtered measurement error $E_{\text {sys }}(T)$, the residual error $\tilde{E}(T)$, depicted in Fig. 9, shows the susceptibility to measurement noise. It can be evaluated by the standard deviation $\sigma_{\mathrm{E}}$. Note that outliers due to bubbles should be suppressed, if only the robustness against noise during normal operation is of interest. The associated histogram in Fig. 9 indicates that the $\mathrm{AWP}_{\mathrm{PCA}}$ is less susceptible to noise than Roosnek's method. This can be explained, if the

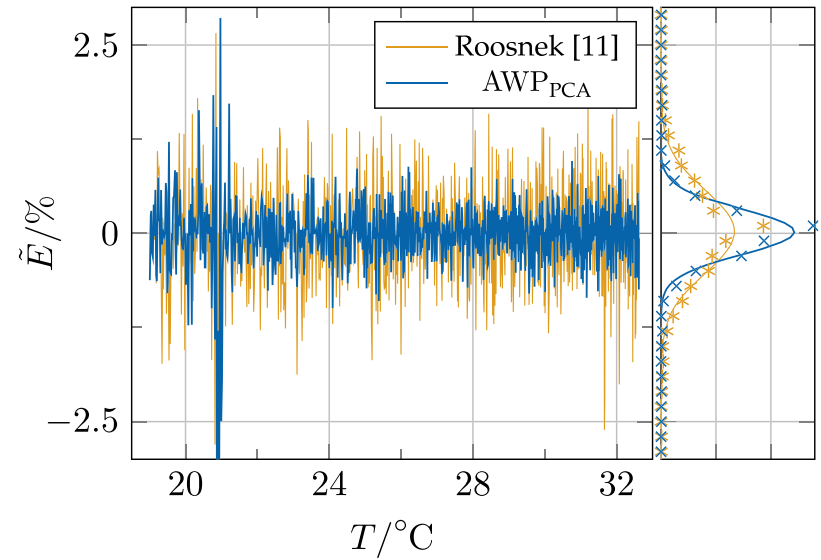

Figure 9: Measurement errors of the AWP method and Roosnek's method over the temperature with compensated trend (left) and their associated histograms with fitted Gaussian distributions (right).

possible sources of the measurement noise are examined: electronic noise and turbulence [20]. Roosnek's method calculates the TTD using a linear fit in the phase domain, whereby one period of the measurement signal is considered in the estimation. In contrast, $\mathrm{AWP}_{\mathrm{PCA}}$ combines several AWP coefficients, leading to a larger time range considered in the estimation and a higher robustness against electronic noise. Furthermore, Figure 5 shows that $\mathrm{AWP}_{\mathrm{PCA}}$ uses information from the first reflection additionally to the direct propagation path, which results in a spatial averaging. Due to the limited spatial extension of the vortices in the turbulent flow, this spatial averaging can also explain a higher robustness against turbulence.

As a calibration over the whole temperature range is expensive, the influence of using only a small part of the calibration set is investigated in Fig. 10. For this investigation, instead of using all 19 temperature steps, only the steps $\left[T_{1}, T_{i}\right]$ were used for the calibration, whereas the measurement error (26) was calculated on the whole test set $\mathcal{T}$ to evaluate the maximal systematic error (27). It is observable that repeating the flow sequence (24) for two temperature steps to get a calibration set is sufficient to improve the systematic error compared to Roosnek's method. The choice of the AWP scale has little to none influence on this result.

To verify that the AWP methods also perform well, independently of the mixture of interfering signals and fluid signals, the systematic error and the noise robustness are evaluated on the data set of the transducer pair T3/T4. Table 1 and 2 list the robustness against noise and against interfering signals for the two transducer pairs and three reasonable AWP scales. Compared against Roosnek's 


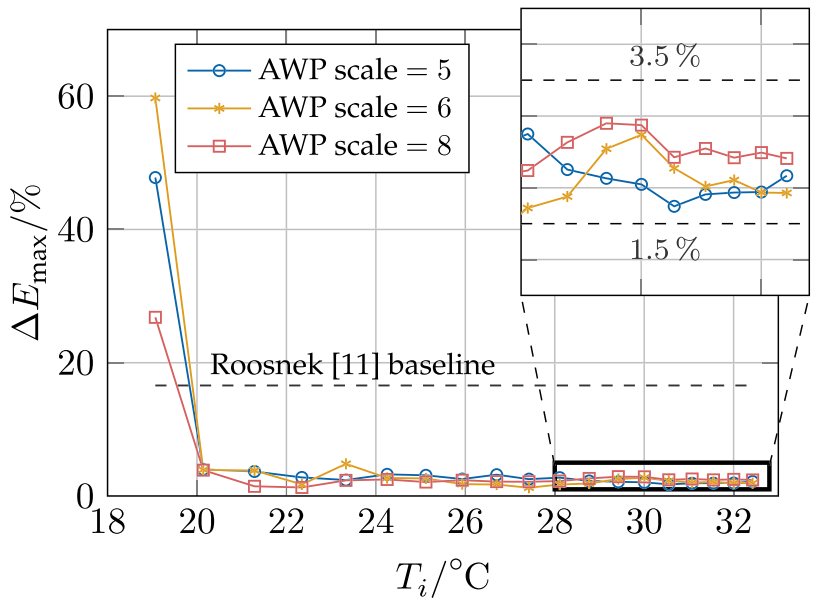

Figure 10: Maximal systematic error (27) of the $A W P_{P C A}$ for three different AWP scales when using only a subset $T \in\left[19^{\circ} \mathrm{C}, T_{i}\right]$ of the calibration set.

Table 1: Noise induced standard deviation of the measurement fluctuation for different paths and methods calculated by (28).

\begin{tabular}{lrrrr}
\hline Set & AWP scale & Roosnek [16] & AWP $_{\text {Max }}$ & AWP $_{\text {PCA }}$ \\
\hline \multirow{3}{*}{$\mathrm{T} 1 / \mathrm{T} 2$} & 5 & $0.75 \%$ & $0.68 \%$ & $0.55 \%$ \\
& 6 & $0.75 \%$ & $0.74 \%$ & $0.59 \%$ \\
& 8 & $0.75 \%$ & $0.69 \%$ & $0.59 \%$ \\
\multirow{2}{*}{$\mathrm{T} 3 / \mathrm{T} 4$} & 5 & $0.70 \%$ & $0.73 \%$ & $0.54 \%$ \\
& 6 & $0.70 \%$ & $0.72 \%$ & $0.55 \%$ \\
& 8 & $0.70 \%$ & $0.74 \%$ & $0.58 \%$ \\
\hline
\end{tabular}

Table 2: Systematic measurement error for different paths and methods calculated by (27).

\begin{tabular}{lrrrr}
\hline Set & AWP scale & Roosnek [16] & AWP $_{\text {Max }}$ & AWP PCA $_{\text {PC }}$ \\
\hline \multirow{3}{*}{$\mathrm{T} 1 / \mathrm{T} 2$} & 5 & $16.59 \%$ & $6.51 \%$ & $2.17 \%$ \\
& 6 & $16.59 \%$ & $4.77 \%$ & $1.93 \%$ \\
& 8 & $16.59 \%$ & $5.97 \%$ & $2.41 \%$ \\
\multirow{2}{*}{$\mathrm{T} 3 / \mathrm{T} 4$} & 5 & $3.81 \%$ & $4.91 \%$ & $1.90 \%$ \\
& 6 & $3.81 \%$ & $4.09 \%$ & $1.35 \%$ \\
& 8 & $3.81 \%$ & $7.83 \%$ & $2.25 \%$ \\
\hline
\end{tabular}

method, the systematic error $\Delta E_{\max }$ is improved from $3.8 \%$ to $1.4 \%$ for the transducers T3/T4 and from $16.6 \%$ to $1.9 \%$ for $\mathrm{T} 1 / \mathrm{T} 2$, where the level of interfering signals is significantly higher than for T3/T4. Using (28) as the criterion for the robustness against noise, the $\mathrm{AWP}_{\mathrm{PCA}}$ achieves an average improvement of $0.15 \%$.

\section{Conclusion}

The application of analytic wavelet packets to the transittime difference estimation in ultrasonic flow measure- ments has been presented. For this purpose, a calibration set has been used to identify coefficients in the timefrequency domain that strongly correlate with the observable. Furthermore, a weighting scheme based on the principal component analysis that allows the information fusion of the selected time and frequency ranges was introduced.

After the description of the calibration and the associated estimation algorithm, the method was evaluated in a clamp-on flow measurement system with two transducer pairs and compared to the method presented by Roosnek [16]. In this evaluation, special attention was paid to the robustness against interfering signals and measurement noise.

The results have shown that the robustness against both interfering signals and noise could be improved compared to Roosnek's method on all measurement sets. Furthermore, the investigation of different calibration sets has shown that it is sufficient, if the flow velocity goes through two cycles with slightly different temperatures. A calibration set over the whole temperature range is not necessary.

The extension of the proposed method to estimate absolute transit-times and to work without calibration will be part of future research.

\section{References}

1. G. Andria, F. Attivissimo, and N. Giaquinto. Digital signal processing techniques for accurate ultrasonic sensor measurement. Measurement, 30 (2): 105-114, 2001.

2. J. Beyerer, M. Richter, and M. Nagel. Pattern Recognition: Introduction, Features, Classifiers and Principles. Walter de Gruyter GmbH \& Co KG, 2017.

3. I. Daubechies. Ten lectures on wavelets. Regional conference series in applied mathematics. Society for Industrial and Applied Mathematics, Philadelphia, Pa., 1992.

4. C. Guetbi, D. Kouame, A. Ouahabi, and J. P. Chemla. Methods based on wavelets for time delay estimation of ultrasound signals. In 1998 IEEE International Conference on Electronics, Circuits and Systems. Surfing the Waves of Science and Technology, volume 3, pages 113-116, 1998.

5. U. Hempel, S. Wöckel, and J. Auge. Ultraschallbasierte informationsgewinnung in der verfahrenstechnik. Chemie Ingenieur Technik, 82 (4): 491-502, 2010.

6. M. Hua, W. Hui, and L. Mingwei. High-precision flow measurement for an ultrasonic transit time flowmeter. In 2010 International Conference on Intelligent System Design and Engineering Application, volume 1, pages 823-826, 2010.

7. S. A. Jacobson, P. N. Denbigh, and D. E. H. Naudé. A new method for the demodulation of ultrasonic signals for cross-correlation flowmeters. Ultrasonics, 23 (3): 128-132, 1985. 
8. N. Kingsbury. Design of q-shift complex wavelets for image processing using frequency domain energy minimization. In Proceedings 2003 International Conference on Image Processing, volume 1, pages 1013-1016, 2003.

9. W. T. Kuang and A. S. Morris. Using short-time fourier transform and wavelet packet filter banks for improved frequency measurement in a doppler robot tracking system. IEEE Transactions on Instrumentation and Measurement, 51 (3): 440-444, 2002.

10. M. Kupnik, E. Krasser, and M. Gröschl. Absolute transit-time detection for ultrasonic gas flowmeters based on time and phase domain characteristics. In 2007 IEEE Ultrasonics Symposium Proceedings, pages 142-145, 2007.

11. L. C. Lynnworth and Y. Liu. Ultrasonic flowmeters: Half-century progress report, 1955-2005. Ultrasonics, 44 (Suppl. 1): e1371-e1378, 2006.

12. E. Mandard, D. Kouame, R. Battault, J.-P. Remenieras, and F. Patat. Methodology for developing a high-precision ultrasound flow meter and fluid velocity profile reconstruction. IEEE Transactions on Ultrasonics, Ferroelectrics, and Frequency Control, 55 (1): 161-172, 2008.

13. V. Matz, M. Kreidl, and R. Smid. Classification of ultrasonic signals. International Journal of Materials, 27(3): 145-155, 2006.

14. N. Michalodimitrakis and T. Laopoulos. On the use of wavelet transform in ultrasonic measurement systems. In IMTC 2001. Proceedings of the 18th IEEE Instrumentation and Measurement Technology Conference. Rediscovering Measurement in the Age of Informatics, volume 1, pages 589-594, 2001.

15. E. Oruklu, N. Jayakumar, and J. Saniie. Ultrasonic signal compression using wavelet packet decomposition and adaptive thresholding. In 2008 IEEE Ultrasonics Symposium, pages 171-175, 2008.

16. N. Roosnek. Novel digital signal processing techniques for ultrasonic gas flow measurements. Flow Measurement and Instrumentation, 11 (2): 89-99, 2000.

17. S. J. Rupitsch. Piezoelectric Sensors and Actuators. Springer-Verlag Berlin Heidelberg, Heidelberg, 2018.

18. I. W. Selesnick, R. G. Baraniuk, and N. G. Kingsbury. The dual-tree complex wavelet transform. IEEE signal processing magazine, 22 (6): 123-151, 2005.

19. G. Serbes, N. Aydin, and H. O. Gulcur. Directional dual-tree complex wavelet packet transform. In 2013 35th Annual International Conference of the IEEE Engineering in Medicine and Biology Society (EMBC), pages 3046-3049, 2013.

20. T. Strunz, A. Wiest, A. Fleury, and T. Fröhlich. Influence of turbulence on ultrasonic flow measurements. In 5th IGHEM Conference, 2004.

21. K. Tawackolian, O. Büker, J. Hogendoorn, and T. Lederer. Investigation of a ten-path ultrasonic flow meter for accurate feedwater measurements. Measurement Science and Technology, 25: 075304, 2014.

22. T. Weickert, C. Benjaminsen, and U. Kiencke. Analytic wavelet packets-combining the dual-tree approach with wavelet packets for signal analysis and filtering. IEEE Transactions on Signal Processing, 57 (2): 493-502, 2009.

23. H. Zhang, C. Guo, and J. Lin. Effects of velocity profiles on measuring accuracy of transit-time ultrasonic flowmeter. Applied Sciences, 9 (8): 1648, 2019.

\section{Bionotes}

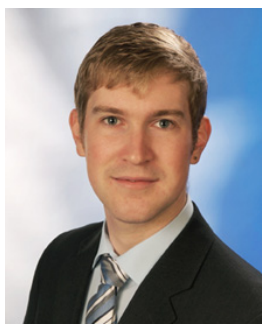

\section{Matthias Bächle}

Karlsruhe Institute of Technology, Institute of Industrial Information Technology, Karlsruhe, Germany matthias.baechle@kit.edu

Matthias Bächle obtained his bachelor's and master's degree in Electrical Engineering/Information Technology at the Karlsruhe Institute of Technology, Germany in 2014 and 2016, respectively. He is currently working as a research associate at the Institute of Industrial Information Technology (IIIT) at the Karlsruhe Institute of Technology, Germany, where he is pursuing his Ph. D. degree. His current research interests include signal processing techniques and their application to flow measurement using transit-time of ultrasonic pulses.

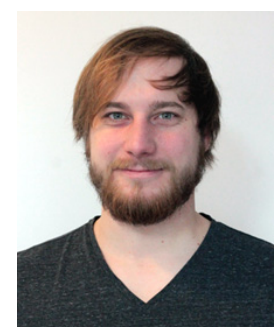

\section{Daniel Alexander Schwär}

Karlsruhe Institute of Technology, Institute of Industrial Information Technology, Karlsruhe, Germany daniel.schwaer@kit.edu

Daniel Schwär received his bachelor's degree in Electrical Engineering/Information Technology at the Karlsruhe Institute of Technology (KIT), Germany in 2016. He is currently doing his master's degree also in Electrical Engineering/Information Technology at the KIT with the focus on Signal processing. His master thesis was about flow measurement with wavelet transform at the Institute of Industrial Information Technology (IIIT).

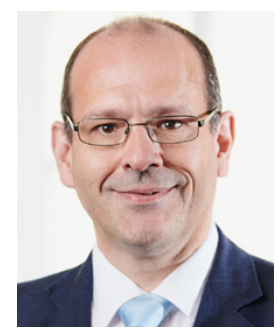

\author{
Fernando Puente León \\ Karlsruhe Institute of Technology, Institute \\ of Industrial Information Technology, \\ Karlsruhe, Germany \\ fernando.puente-leon@kit.edu
}

Fernando Puente León is a Professor with the Department of Electrical Engineering and Information Technology at Karlsruhe Institute of Technology, Germany, where he heads the Institute of Industrial Information Technology (IIIT). From 2001 to 2002, he was with DS2, Valencia, Spain. From 2002 to 2003 , he was a postdoctoral Research Associate with the Institut für Mess- und Regelungstechnik, University of Karlsruhe. From 2003 to 2008, he was a Professor with the Department of Electrical Engineering and Information Technology, Technische Universität München, Germany. His research interests include image processing, automated visual inspection, information fusion, measurement technology, and pattern recognition. 